$$
\begin{aligned}
& \therefore \\
& \text { JAN: : } 1933
\end{aligned}
$$

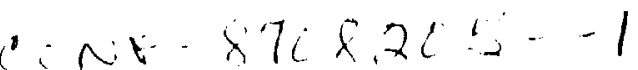

LA-UR- $-87-4077$

DE88 004313

\title{
TITLE: A LECTURE ON DETONATION-SHOCK DYNAMICS
}

\section{AUTHOR(S) D(onald $S(\cot t)$ Stewart \\ $J$ (ohn) B(ohdan) Bdzil}

\section{DISCLAIMER}

\begin{abstract}
This report was prepared as an hccount of work sponswred by an agency of the linited Siates Government Netther the I/nited States (iovernment nor any agency thereof, nor any of their employees, makes any warranty, express or implied, or assumes any legal liability or responsihility for the acciracy. completeness, or usefulness of any information, apparatus, product, or prexess disclessed, or represents that its use would not infringe privately owned rights Reierence herein 10 any specific commercial product, proxess, or service hy trade name, Irademark. manufacturer. or otherwise dex nut necessarily conssitute or imply its endorsement, recom. mendation, or fuvoring by the I/nited States (iovernment or any apency thereof The views and opinoons of authors expressed herein do not necessurily state or reflect those of the linited States Government or any apency thereof.
\end{abstract}

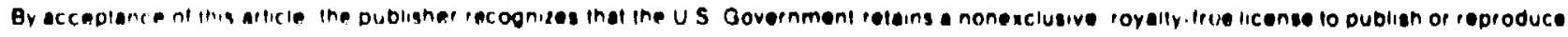

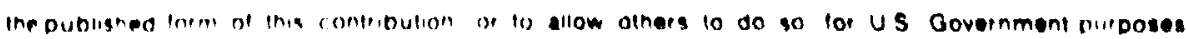

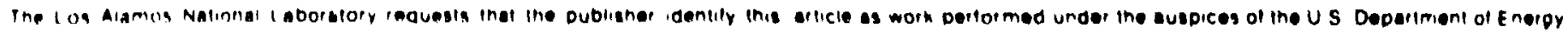




\section{A Lecture on Detonation-Shock Dynamice}

D. S. Stewart

University of Dlinois, Urbans, Ilinois, 61801

J. B. Bdril

Los Alamos National Laboratory, Los Alanos, New Mexica 87545

\section{Abstract}

We summarize some recent developments of J. B. Bdzil and D. S. Stewart's investigation into the theory of multi-dimensional, time-dependent detonation. These advances have led to the development of a theory for describing the propagation of high-order detonation in condensed-phase exploaives. The central approximation in the theory is that the detonation shock is weakly curved. Specifically, we assume that the radius of curvature of the detonction shock is large compared to a relevant reaction-zone thickness.

Our main findings are: (1) the flow is aussi-steady and pearly one dimensional along the normal to the detonation shock, and (2) the small deviation of the normal detonation velocity from the Chapman-Jouguet (CJ) value is generally a function of curvature. The exact functional form of the correction depends on the equation of state (EOS) and the form of the energy-release law.

\section{Introduction}

In this lecture we will deacribe a theory for unsteady, unsupported, multi-dimensional detonation propagation for the standard exploaive model; the reactive Euler equation for a preacribed EOS and rate law. For this model, the detonation structure is ZND, i.e., a shock followed by a reaction zcne which contains an embedded, trailing sonic locur. See Figure 1. In laboratory frame coordinates, the governing equations for this model are

$$
\frac{D \rho}{D t}+\rho(\nabla \cdot u)=0
$$




$$
\begin{gathered}
\rho \frac{D u}{D t}=-\nabla P \\
\frac{D E}{D t}+P \frac{D(1 / \rho)}{D t}=0 \\
\frac{D \lambda}{D t}=r,
\end{gathered}
$$

where in the above $\rho, u, P, E, \lambda$ and $r$ are respectively the density, particle velocity, pressure, epecific internal energy, single reaction progress variable and the rate of forward reaction. To complete the specification of the problem we need to choose constitutive relations for the internal energy function $E(P, \rho, \lambda)$ and the rate law $r(P, \rho, \lambda)$. For illustrative purposes we select the polytropic form for $E$,

$$
E=\frac{P}{\rho}(\gamma-1)^{-1}-q \lambda
$$

where $\gamma$ is the polytropic exponent, and $q$ is the sperific heat of reaction. The solution of these enuations must satisfy the standard normal shock relations at the leading detonation shock.

The theoretical developments are carried out in the limit that the radius of curvature of the shock front (R) is much greater than a characteriatic reaction-zone length ( $\left.r_{l}\right)$, i.e.

$$
\delta^{2}=\left|r_{\ell}\right| R \mid<<1
$$

With appropriate assumptions, the main result is that the velocity of the leading detonation shock along its normal deviates from the Chapman-Jouguet value by amall amount that is proportional to curvatire (in the aimpleat casen) and more generally is a function of curvature, i.e.

$$
D_{n}=D_{C J}-\alpha \kappa \text { where } \alpha=\text { constant or } \alpha=\alpha(\alpha)
$$

We were led to the discovery of (i), by our vesire to formulate a rigorous theory of the evolution of the detonation shock in complex, two-dimensional (2D) and three-dimensional 
(3D) geometries, which retained full reaction-zone effects, time dependence, and which was a physically correct and simple-to-use method for correcting detonation velocity. This study was aimed at gaining a fundamental understanding of multi-dimensional detonation.

Our theory is closely reiated to Whitham's theory of Geometrical Shock Dynamics [1]. Similarly. our theory streases the dynamics of the shock. However, unlike Whitham. we have a systematic theory of the following flow that supports the shock that is strictly valid when the radius of curvature is large compared to the reaction-zone length.

In Section 2, we give a brief history of earlier developments in 2D dctonation theory We sketch the fundamental approximations and our recent theoretical developments, in Sec:ion 3. In Section 4, we give some examples of fundamental desonation interactions, while in Section 5. we extend or modeling by examining an energy-ielease rate that is strongly dependent on state. Finally in Section 6. we comment on the practical implications of the theory for explosive engineering.

\section{History of the development}

The line of the development of the research prasented here can be traced back through the work of Wood and Kirkwood [2] in 1954. Bdzil [3] in 1981. and through the recent collaboration of Bdzil and Stewart from 1984 to present. See references !4| and $|5|$.

The fact the detonation propagation speed is dramatically affected by diverging geometry is illustrated by a standard experiment in a rate stick. In that experiment. a cylindrical stick confined by an inert tube is ignited ot the bottom by means of a planewave explosive lens and a pad of high pressure booster explosive $A$ nominally plane, overdriven detonation is thus introduced at the bottom of the stick. As time passes. the detonatior shock in the stick becomes curved. because the high-pressure flow expands the iube wallo into 
the relative vacuum sur:ounding the experiment (i.e., room pressure air). As a result, the plane character of the wave is destroyed. When a steady detonation develops in the atick it has an elliptical-like shape. The final steady 2D-detonation velocity can be measured by simple means and is found to be a function of the radius of the stick and the degree of confinement, i.e., tube wali material and thickness. The steady detonation velocity is reduced irom the 1D Chapman-Jouguet value, $D_{C J}$, by an amount that becomea greater as the radius of the stick. $\boldsymbol{R}_{\text {o, }}$ is reduced (see Figure 2 for a schematic diagram). At some critical radius, experiments using witness plates show that a steady detonation is not propagated in the stick Presumably some form of extinction occurs.

Tine first theoretical calculations that explained these experimentally observed effects were carried out by Wood and Kirkwood [2]. They used the basic model described in the introduction specialized to a steady, radially symmetric tow. By restricting their analysis to the central streamline. and by further assuming that the 2D radial fow divergence, $\nabla$, was known, they reduced the problem to a system of nonlinear ordinary-differential equations for the steady detonation structure. In particular, they assumed that the quantity. $F$ u was related to a single ad hoc parameter (e.g.. $R$ ) that measures the divergence of the flow In these equations the detonation velocity. $D$. is an unknown constant parameter and $R$ is a specified parameter. Fickett and Davis $|6|$ further showed that this system of equations could be reduced to " single equation for $U^{2} \equiv|u-D|^{2}$. the kinetic energy in the main flow direction, a a function of the reaction progress variable $\lambda$.

A qualitative analysis of this governing equation can be carried out quite corveniently in the $\left(U^{2}, \lambda\right)$-phase plane $A$ given value of $D$ defines the atarting value for $U^{2}$ at the shock. The task is to determine an integral curve in this plane. that follows $U^{2}$ as $\lambda$ changes from $\lambda=0$ at the shock to $\lambda=1$ at complete reaction. In the limit that the flow 
divergence is zero, the integral curve terminates at a singular point at $\lambda=1$. When the flow divergence is non sero, an additional singular point is found in the phase plane that corresponds to the intersection of the tnermicity line and the sonic line. The reaction is incomplete ot this new saddle-type singular point. The integral curve will pass through this point, for only a single value of $D$ for a given $R$, i.e., $D(R)$. In general, this relationship must be found by numerical shooting techniques. An excellent account of the details of th is work is found in Fickett and Davis's book (1979) [6], Section 5g3.

The next contribution to the development of the current theory is due to $\mathrm{Bdzil}[3]$. He analyzed the piobiem of a steady-state $2 \mathrm{D}$ detonation in rate-stick geometry. This analysis was rigorous and not ad hoc as was that of Wood and Kirkwood. It was not restricted to the central streamline, but considered the entire 2D problem. This theory is an asymptotic theory which is consistent with the assumption that the stick radius, $R_{a}$, is large compared to a $1 \mathrm{D}$ reaction-zone length. Once again a parameter equivalent to

$$
\delta^{2} \equiv\left|r_{2} / R_{0}\right|<<1 \text {, }
$$

can be defined. (In Bdzil's account $\delta$ is related cirectly to th, angle of the streamline deflecticin at the confinement boundary.) This assumption is equivalent to a small shock slope, with an $O(1)$ change in the shock position $Z_{2}$ (measured on the scale o. reactionzone lengths) taking place over the lateral distance scale $r \delta \sim O(1)$ (manj- reaction-zone lengths).

Bdzil found that all the leading features of the flow could be determined, and that they were carameterized by the shock locus function, $Z$, In turn, the shock locus was a f. 1 nction of the scaled transverse coordinate $\varsigma=r \delta$ and. for a pasticular example involving the choice of EOS and rate law, satisfied the second-order ordinary-differe utial equation

$$
\frac{D_{C}}{2}\left[\frac{d Z_{0}}{d \zeta}\right]^{2}=u \frac{d^{2} Z_{0}}{d s^{2}}-D^{(\Delta)} .
$$


where $D^{(2)}$ is identified by the expansion

$$
D=D_{C J}+\delta^{2} D^{(2)}
$$

and measures the deviation of the steady detonation velocity from $D_{C J}$.

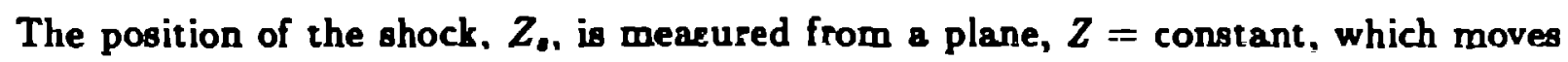
with the steady detonation velocity, $D$. The function $Z_{\bullet}(s)$ determines the local detonation velocity normal to the shock along its extent. Indeed, even though this is not made explicit in Bdzil's paper, equation (8) is equivalent to the ccordinate-independent statement

$$
D_{n}=D_{C \cdot J}-a \kappa+o(\kappa)
$$

where $D_{n}$ is the velocity along the shock normal. In the above. $\alpha$ is a constant (the assumptions about the EOS and rate law in [3] give $\alpha$ a specific value).

In 1984 we started work on the simf.lest, most straightforward extension of this steady theory that would include time dependence. We noticed that in order to include time dependence in a quasi-steady theory, it was necessary to introduce a slow-time scale such that the time dependence entered the theory at the same order as the shock curvature. In particular if on the reaction-zone length scale the shock locus, $Z_{1}$, is an $O(1)$ function, chen the relevant slow-time scale is

$$
r=\delta^{2} t
$$

where $t$ is meagured with the reaction-zone time scale. Cakulations with these scaling assumptions show that at leading order, the flow through the reaition zone has the same form as it does in the steady-state problem. 1.e., it is quast-steady However, the shock locus. which in what parameterizes the solution. is now a function of both the scaled transverse coordinates and the scaled tirie $r$ 
In contrast to (8), the shock locu, $Z_{0}$, now satisfies the partial-differential equation

$$
\frac{\partial Z_{s}}{\partial \tau}-\frac{D_{C J}}{2}\left[\frac{\partial Z_{S}}{\partial \varsigma}\right]^{2}=\alpha \frac{\partial^{2} Z_{0}}{\partial \varsigma^{2}}-D^{(2)} \text {, }
$$

where $Z_{a}$ is measured from a constant velocity plane. The above equation is a nonlirear heat equation. Indeed for $\alpha=$ constant, equation (11) can be reduced to a Burgers' equation for the shock slope, $\partial Z_{\bullet} / \partial \zeta$. On these length and time scales $\zeta$ and $r$, the evolution of the shock is not governed by a hyperbolic equation, but by the parabolic equation (11). A natural question to ask is why do we find a parabolic evolution equation for a system of hyperbolic equations?

The answer is found in Bdzil and Stewart's $[4]$ (1986) paper on time-dependent 2D detonation. In that paper, we studied the transients that carry an initially $1 D$ detonation into a steady-state 2D detonation. In the example we considered, an initially steady 1D detonation encounters an unconfined corner in the explosive (see Figure (3a)). After the wave reached the corner, the explosive products expanded into the vacuura and the detonation shock began to curvc. Because the problem is hyperbolic, a traveling wave head was defined on the detonation shock to the left if which tinere was no disturbance of the $1 \mathrm{D}$ detonation.

We selected the exprosive EOS and rate law with the goal of achieving a ID detonation that was linearly stable to both transverse and flow-direction disturbances. With this goal in mind, we adopted a polytropic EOS model and a rate law for which most of the chemical heat release is given up immediately behind the shock. Thu was followed by a smaller resolved heat release that tnok place over a finite distance behind the shock and which controled the dynamics of the problem. For this "small resolved heat-release model." the dynamics of the ID detonation occur on the "fast" time scale $\delta t$. Our results showed that disturbances on the shock propagate according to a hierarchy of two distinct flow regions 
which occur on the time scales $\delta t$ and $\delta^{2} t$.

In the first region the displacement of the shock is small and the dynamics, which occur on the $\delta t$ time scale, is wave-like (hyperbolic). This region contains the hydrodynamic wave head, i.e., the leftmost point of the shork disturbance. The magnitude of the shock displacement, length and time scales for this region are given by

$$
Z, \sim O(\delta) \text {, ith } \delta^{1 / 2} r, \delta t
$$

The second region is a diffusion-like region (parabolic). In this region the shock dislacement from plane is the largest and the disturbance extends over both the greatest length and time scales. The magnitude of the shock displacement. length and time scales for this region are given by

$$
Z_{.} \sim O(1) \text { with } \delta r, \delta^{2} t
$$

Figures $3 a$ and $3 b$ shows a schematic diagram of both the initia' configuration and the evolutionary phase of the detonation shock for these two regions.

What we learned from [4] is that the parabolic flow is naturally embedded in the hyperbolic system. The hyperbolic region while defining the wave head of the disturbance is associated with a small ainplitude shock deflection. In contrast the parabolic region is associated with a large scale shock deflection and is the most important region to characterize and measure. The advantage of this description is the relative simplicity of the parabolic region. which involves at most the solution of a gimple seccnd-order partial-differential equatiun (the nonlinear heat equation) Additionally, practical experience with the technologically important case of condensed phase propellants and explosives shows that they have broad well defined detonation shocks. To check the validity of the steady theory for condensed phase expiosives, Engelke photographed the shock loci and compared them with 
the predictions of the steady theor. See Bdzil $[3 \mid$ and Engelke and Bdzil $[7]$. The theory and experiment were shown to be in qualitative and even quantitative agreement. Therefore, consistency of the unsteady and steady theories then also argues for the parabolic scales.

The results of $[4]$ confirmed the importance of evolution equations of the parabolic type which were discovered earlier. The earlier work was eventually recorded in a paper by Stewart and Bdzil [5], where some examrles of relationships between the norma] detonation-shock velocity and the curvature were derived for the first time.

The simplicity of the parabolic description makes it possible to do routine calculations of a class of unsteady detonation problems. The detonation-wave spreading problems of greatest interest occur in explosives with complicated shapes. If we are to apply the parabolic description outlined above to such problems, we need to carry out the analysis in a system of intrinsic (or problem determined) coordinates. These calculations are the subject of the next section.

\section{Sketch of the analysis}

In this section we sketch the analysis and explain the approximations used in deriving the shock-evolution equation and the flow description. The model equations are the reactive Euler equations, subject to the shock Hugoniot conditions for a specific EOS and rate law. The presentation here is an outline of the more detailed discussion found in Bdzil and Stewart $[8 \mid$.

The coordinates we choose are shock-attached coordinates. and the problem is three dimensional. Here $\xi_{1}$ represents arc length along the shock in the directions of the principle curvatures $(s=1,2)$ defined by the ingtantaneous shocl: surface. The variable $n$ represents 
the distance normal to tlie shock. The coordinates $\xi_{1}$ and $n$ form a locally orthogonal coordinate system. A picture of the intrinsic-coordinate system for 2D is shown in Figure 4. Because we have chosen an intringic-coordinate system, the shock curvature appears explicitly in the governing equations of motion. These equations becume

Mass:

$$
\rho_{. z}-\left[\rho\left(D_{n}-u_{n}\right)\right]_{. n}+\kappa \rho u_{n}+\ldots=0,
$$

Energy:

$$
E_{. t}-\left(D_{n}-u_{n}\right) F_{. n}-\left(P / \rho^{2}\right)\left[\rho_{t}-\left(D_{n}-u_{n}\right) \rho_{. n}\right]+\ldots=0 \text {, }
$$

Momentum

$$
\begin{array}{ll}
n: & u_{n, 1}+\left(D_{n}-u_{n}\right) \dot{I}_{n, n}+(1 / p) P_{. n}+\ldots=0, \\
\xi_{1}: & u_{\xi_{1}:}-\left(D_{n}-u_{n}\right) u_{\xi_{n}, n}+\ldots=0, \quad \imath=1,2
\end{array}
$$

Rate:

$$
\lambda_{t}-\left(D_{n}-u_{n}\right) \lambda_{. n}=r+\ldots
$$

Note that $D_{n}$ is the instantaneous shosk relocity along the shock normal, $u_{n}$ and $u_{\varepsilon}$ are laboratory-frame particle velocities in the $n$ and $\xi_{2}$-directions respectively. The curvature that appears in the above equations is the sum of the principal curvatures, $\kappa \equiv \kappa_{1}+\kappa_{2}$. Higher order terms in :he shock curvature are indicated by ellipses.

To these equations we add the shock relations

$$
\begin{gathered}
\rho_{-} D_{n}=\rho_{-}\left(D_{n-u_{-}}\right), \quad P_{-}=\rho_{-} u_{n_{+}} D_{-}, \quad \lambda_{+}=0 . \\
\frac{D_{n}^{2}}{2}=E_{-}+\frac{P_{-}}{\rho_{-}}+\frac{1}{2}\left(D_{n}-u_{n_{-}}\right)^{2}, \quad u_{n_{-}}=0.2=1.2 .
\end{gathered}
$$

The minus subscript refers to the state ahead of the shock, the plus subscript refers to the state behind the shock. In these relations we have adopted the strong shock approximation and have set terms proportional to $P_{-}$to zerc (we have anticipated that $E_{-} \sim P_{-} \rho_{-}$).

We make the explicit assumption that the curvature is

$$
\kappa \equiv \delta^{2} \bar{\kappa}, \quad \delta^{2}<1 .
$$


where $\hat{\kappa}$ is the scaled shock curvature and $\delta^{2}$ measures the magnitude of curvature relative to the $1 \mathrm{D}$ reaction-zone length. The length and time scales required are

$$
r=\delta^{2} t, \quad n, \text { and } \zeta_{i}=\delta \xi_{i}, \text { for } i=1,2 \text {. }
$$

We introduce the formal expansions for the dependent variables

$$
\begin{aligned}
& u_{n}=u_{n_{0}}^{(0)}+\delta^{2}{\nu_{n}}_{n}^{(2)}+\ldots, u_{\zeta_{1}}=\delta^{2} u_{\varsigma_{1}}^{(2)}+\ldots \\
& P=P^{(0)}+\delta^{2} P^{(2)}+\ldots, \rho=\rho^{(0)}+\delta^{2} \rho^{(2)}+\ldots \\
& \lambda=\lambda^{(0)}+\delta^{2} \lambda^{(2)}+\ldots, D_{n}=D_{C J}+\delta^{2} D_{n}^{(2)}\left(\zeta_{1}, \tau\right)+\ldots
\end{aligned}
$$

Using these expansions in equaticns (12) - (16) we find that through and including $O\left(\delta^{2}\right)$, the equations that govern the flow reduce exactly to the equations for quasi-steady flow in cylindrical geometry

$$
\begin{gathered}
-\left[\rho\left(D_{n}-u_{n}\right)\right]_{. n}+\kappa \rho u_{n}+\ldots=0, \\
\left(D_{n}-u_{n}\right) E_{. n}-\left(P / \rho^{2}\right)\left[\left(D_{n}-u_{n}\right) \rho_{. n}\right]+\ldots=0 \\
\left(D_{n}-u_{n}\right) u_{n}+(1 / \rho) P_{n n}+\ldots=0, \\
\left(D_{n}-u_{n}\right) u_{\xi_{0}, n}+\ldots=0,8=1,2 \\
-\left(D_{n}-u_{n}\right) \lambda_{n}=r+\ldots .
\end{gathered}
$$

since from equation (24) and the shock conditions it follows that $u_{\varepsilon}=0$.

In Section 2 we mentioned that Wood and Kirkwood $|2|$ treated the central streamline problem. Equations (21) - (2b) taken together with the normal shock relat inns are equivalent to the problem they treated. Now, the terms due to the flow divergence are rigorously identified as being proportional to the local shock curvature. $\kappa$. The above problem .hen admits an eigenvalue detonation as its solution. As Wood and Kirkwood showed, it defines a relation between the two parameters $D_{n}$ ard $x$ as a condition necessary for the integral 
curve in the $\left(u_{n}^{2}, \dot{\lambda}\right)$-piane to pass through the sadale singular point, where the flow is sonic. Generally speaking, we have the requirement that there exists a relation of the form

$$
D_{n}=D_{n}(\kappa)
$$

To illustrate this point we give the equation. Let $U_{n} \equiv u_{n}-D_{n}$, and consider the polytropic EOS

$$
E=\frac{P}{\rho}(\gamma-1)^{-1}-q \lambda
$$

Straightforward manipulation of equations (21) - (25) yields the single ordinary-differential equation for $U_{n}^{2}$ in terms of $\lambda$, namely

$$
\frac{\left.\ddot{i} U_{n}^{2}\right)}{d \lambda}=\frac{2 U_{n}^{2}\left\{q(\gamma-1) r-c^{2}\left(D_{n}+U_{n}\right) \kappa\right\}}{r\left(c^{2}-U_{n}^{2}\right)} \text {, }
$$

where the sound speed is given by $c^{2}=\gamma P / \rho=(\gamma-1)\left[\left(D_{n}^{2}-U_{n}^{2}\right) / 2+q \lambda\right]$. The shock boundary condition requires that

$$
U_{n+}=-\frac{D_{n}(\gamma-1)}{(\gamma+1)}
$$

Following the ncmenclature of Fickett and Davis, the ( )-term in the numerator of (28) defines the thermicity locus in the $\left(U_{n}^{2}, \lambda\right)$-plane, and $\left(c^{2}-U_{n}^{2}\right)$ defines the sonic locus. These curves, slong with $r=0$, define the seperatrices and their intersections define the sungular points in the phase plane. The object in the phase plane is to find the integral curve that starts from the shock value given by (29) and terminates at complete reaction. Typical:'; sucin curves must pass through a singular point defined by the intersection of the sonic and thermicity loci. Since $\boldsymbol{\alpha}$ is small. the intersection point is very close to complete reaction. As mentioned before, this point is a saddle To ensure passage through the saddle, condition (26) must hold. 
In order to give a specific form to relationship (26) we must give the rate law. In Stewart and Bdzil [5] it is shown that for the choice

$$
r=k(1-\lambda)^{\nu}, \text { for } 0<\nu<1 \text {, }
$$

equation (26) takes the form

$$
D_{n}=D_{C J}-\alpha \kappa+o(\alpha), \alpha \equiv \frac{\left.\gamma^{2} D_{C J}^{2}\right)^{2}}{k(\gamma+1)^{2}} \int_{0}^{1} \frac{(1+\sqrt{1-\lambda})^{2} d \lambda}{(1-\lambda)^{2}}
$$

For the special case of simple depletion $(\nu=1)$ it can be shown that for diverging geometry $(\boldsymbol{x}>0)$

$$
D_{n}=D_{C J}+\beta \kappa \ln (\kappa)+2 \beta \kappa\left[\ln \left(\beta / D_{C J}\right)-3\right]+\ldots, \beta \equiv \frac{\gamma^{2} D_{C J}^{2}}{k(\gamma+1)^{2}}
$$

\section{Detonation inieractlone}

The formulas given in the last part of Section 3 show that the detoration-shock velocity is a function of the curvature of the shock. In order to describe the evolu cion of the shock we must have a second relation between $D_{n}$ and $\kappa$. Using the surface compatibility ronditions of differential geometry, we liave derived such. a second relation. We call this relation the kinematic-surface condition

$$
\frac{1}{\kappa}\left(\frac{1}{\kappa} D_{n . \zeta}\right)_{. \xi}+D_{n}=-\frac{1}{\kappa}\left(\frac{1}{\kappa} \int_{\xi .}^{\epsilon} \kappa_{. t} d \xi\right)_{.6}
$$

where $\xi^{-}$is a fixed referefice position on the shock (see Figure 4). In 2D, the natural refresentation of the shock locus is in trrme of the angle $\Phi$ that the shock normal makes with respect to a fixed reference direction. Then $\Phi$ is related to the shock curvature by

$$
\phi \equiv \int_{\xi \cdot}^{\xi} \pi d \xi
$$


If we consider the simple case given by equation (31) and use the scalings given by equation (19), we find that equations (31) and (33) imply the following equation for $\phi$,

$$
\phi_{, r}+\frac{D_{C J}}{2} \phi \phi_{, S}=\alpha \phi_{, S s}
$$

Equation (35) is Burgers' equation for $\phi$. The constant a plays the role of viscosity. Burgers' equation has analytical exact solution via the Hopf-Cole transformation and its dynamics have been a tudied extensively. Thus for this example, fundamental shock interaction problems can be studied with these exact solutions. According to our theory, there nuw exists a catalogue of solutions for detonation-shock interactions, that is similar to the catalogue of solutions to Burgers' equation.

Two simple examples from this catalugue are the step-shock solution and the $\boldsymbol{N}$-wave solution to Burgers' equation. The step-shock solution corresponds to the solution for two colliding detonations. providing that the detonating material is large enough that the detonaticn-shock angles are constant in the far field. If two plane detonations are initiated obliquely so as to run into one another, the slope of their common intersected shock locus starts from the left with one value and moves tc another value as we pass to the right. Solutions to Burgers' equation show that ultimately a steady-state step-shock soluticn is attained with a definite shock-shock [1] thickness that depends on $\alpha$. This interaction mimics a reactive Mach stem. Importantly, it is diffuse (see Figure 5a).

The $\boldsymbol{N}$-wave solution corresponds to a positive shock imperfection. In the right and left far field, the detonation is flat and hence $\phi$ is zero In the center the shock is raised. giving rise to an $N$-shape for $\phi$, from left to right. The $N$-wave soiution then shows that this imperfection ultimately "diffuses" away; the time required for "diffusion" of the imperfection depends on the value of $a$ (see Figure 5b) 


\section{Stronger otate dependence of the rate}

The results given by equations (31) and (32) show that the exact functional form of the detonation-shock velocity va curvature relationship depends on the details of the rate law. Bdzil's [3] results, for steady $2 D$ detonation, showed thsi as the sensitivity of the rate to the local state is increased, a steady solution doss not exist when the curvature becomes sufficiently large. This theoretical observation is consistent with experimental observation.

In this section we present a simple model that shows the consequence of increased state sensitivity. Consider the following shock-state dependent rate (sł.ock-state dependence w typical of solid high explosives)

$$
r=k f(\lambda)=\hat{k} \exp \left[-\theta\left(D_{C J}-D_{n}\right)\right] f(\lambda)
$$

Since $D_{n}$ is proportional to the shock pressure, the rate multiplier $k$ is now a function of how hard the particles were hit by the passage of the shock. Individual particles react at a rate that is determined by how hard they were shocked. The fact that the state dependence is sensitive (i.e., large changes in r occur for small changes in $D_{n}$ ), is modeled by requi.ing that the dimensionless parameter

$$
\theta D_{C J}>>1
$$

For the purpose of this illustration. we further consider tne following distinguished limit relating the large parameter $\theta D_{C}$, and $\delta^{2}$

$$
\left[\theta D_{C J}\right]^{-1}=\delta^{2}
$$

U. Ing the expansion for $D_{n}$, the rate law beromes

$$
r=\dot{k} \exp \left[D_{n}^{(2)} / D_{C J}\right] f(\lambda)
$$


Now it is easy to see that for the case $f(\lambda)=(1-\lambda)^{2}$, where $0<\nu<1$, equatio.. (31) still holds, with the exception that $k$ w replaced by $\hat{k} \exp \left[D_{\|}^{(2)} / D_{C J}\right]$. Using the previous definition for acaled curvature, $\pi=\delta^{2} R$, we find the reduced shock velocity curvature relation becomes

$$
-\left(D_{n}^{(2)} / D_{C J}\right) \exp \left[D_{n}^{(2)} / D_{C J}\right]=\hat{\alpha} \hat{k}
$$

where $\hat{\alpha}$ is given by equation (31) for $\alpha$, with $\hat{k}$ replacing $k$. We rewrite equation (40), in order to compare directly with (31) and (32);

$$
D_{n}=D_{C J}-\alpha \kappa \exp \left[-\theta\left(D_{n}-D_{C J}\right)\right]
$$

Frcm equation (41) it is simple to show that for the reduced curvature $\hat{k}$ in the range $0<$ $\hat{x}<\hat{x}_{c r}$, that there are two values for $D_{n}^{(2)}$. Hence the detonation velocity is multivalued for positive (divergent) curvature below a critical value of curvature (see Figure 6). For values of curvature above the critical value, it is not possible to have detonation-shock evolution described by the paratolic scales. A possible consequence of this is extinction of the detonation wave on portions of the curve where the critical curvature is exceeded.

\section{Practical implicatione for exploive englreerlng}

The theory discussed in this lecture pertains to explosive materials in which a brcad, well-defined detonation shock is observed in the limit that the radiue of curvature is large compared to the distance from the leading shock to the anic locus. Indeed this is the case of practical interest for a wide class of explosives.

Engineers who design explosive charges typically use the Huygen's rule of detonation propagation whereby the detonation shoch is advanced along its normal at the coratant Chapman-Jouguet velocity. Our results indicate that this "recipe" should be modified. 
and that the correction factor is generally a function of the curvature. In addition our results show that the detonation structure from shock to sonic locus is easily calculated and is locally a 1D, cylindrical, quasi-steady flow.

The theory then suggests that the $D_{n}(x)$ relation may describe the shock evolution for certain exploaives for a wide range of initial and confinement cr nditions. If this theoretical statement is true, then $D_{n}(\kappa)$ can be determined directly from experiment. For example, $D_{n}(\kappa)$ could be determined from photographs of steady detonation-shork loci in rate sticks. Suppose the steady detonation velocity, $D$, along the axis of the stick has been measured. If $\phi$ is the angle that the shock nonnal (taken from the photograph) makes with the axis of propagation, then the normal velocity is given by

$$
D_{n}=D \cos \phi
$$

The shock curvature $x$ could be inferred from the photograph as well. Thus for the extent of the shock locus shown in the photogiaph, a portion of the $D_{n}(\kappa)$ curve can be constructed

Other experiments, steady or unsteady, in totally different geometries, properly analyzed, should reproduce the samr $D_{\eta}(x)$ in regions of overlap. Consider the case of a ID. unsteady cylindrically or spherically expandir.g detcnation. In this experiment $D_{n}$ is simply $\dot{R}$, the rate of change of the radius from the central point, while $\kappa=1 / R$.

Thus the experimentally determined $D_{n}(\kappa)$ curve, would dctermine the detonation characteristic for many different geometries and configurations without our having detailed knowledge of either the equation of state or the energy-release law

\section{References}

1 Whitham, G B (1971). I.ncar and Nonlinear Waves. Wiley 
2. Wood, W. W. and Kirkwood, J. G. (1954), Diameter effect in condensed explosives: The relation between velocity and radius of curvature in the detonation wave. J. Chem. Phys. 22: 1920-1928.

3. Bdzil, J. B. (1981), Steady-tate two-dimensional detonation, J. Fluid Mech., 108, 195-226.

4. Bdzil, J. B. and Stewart, D. S. (1986), Time-dependent two-dimensional detonation: The interaction of edge rarefactions with finite length reaction zones, J. Fluid Mech., $171,1-26$.

5. Stewart, D. S. and Bdzil. J. B. (1986), The shock dynamics of stable multi-dimensional detonation. University of Illinois, Theoretical and Applied Mechanics Report No. 481, to appear in Combustion and Flame.

6. Fickett, W. and Davis, W. C. (1979), Detonation, University of California Press, Berkeley.

7. Engelke, R. and Bdril, J. B. (1983), A study of the steady-state reaition-zone structure of a homogensous and heterogeneors explosive. Phys. Fluids, 26(5), 1210-1221.

8. Bdzil, J. B. and Stewart, D. S. (1987), Detonation Shock Dynamics, submitted for publication.

\section{Acknowledgments}

An earlier version of this account was written by D. S. Stewart during a visit to the College of Aeronautics under the aupices of AWRE. Aldermaston. Contract No. NNS/32A/ 1A91965. Professor John Clarke's hospitality is gratefully acknowledged. D. S. Stewart is 
supported by a contract with Los Alamos National Laboratory DOE-LANI-9xar-5128c1.

\section{Figure captione}

Figure 1. A schematic representation of the detonation shock with normal and trailing sonic locus displayed.

Figure 2. Rate sticks and the diameter effect. Figures $2 \mathrm{a}$ and $2 \mathrm{~b}$ show schematic diagrams of a standard rate stick experiment. Figure 2a shows the stick prior to initiation. Figure $2 b$ shows steady propagation. Figure $2 c$ shcws the steady value of the detoration velocity $D$ minus $D_{C}$ J plotted versus the inverse of the stick radius, $R_{0}^{-1}$. Twc different cases showing tesu'ts for strong and weak confinement are shown. The open circles show extinction points which indicate no steady propagation for small radius tubes.

Figure 3. Figure 3 a shows the ronfiguration prior to the $1 D$ detonation reaching the vacuum. Figure $3 b$ show: subsequent detonation evolution at two times.

Figure 4. A sketch of the $2 \mathrm{D}$ inirinsic shock-attached coordinate syotem.

Figure 5. Two examples of detenation shock interactions.

Figure 6. Scaled detonation velocity $D_{n}^{(2)} / D_{C}$ J veisus acaled detonation rhock curvature $\mathrm{A}$. 